\title{
Universidades do Brasil, da Espanha e da Itália na Pandemia da Covid-19 numa perspectiva comparada
}

\author{
Universities in Brazil, Spain and Italy \\ in the Covid-19 Pandemic in a comparative perspective \\ Universidades en Brasil, España e Italia \\ en la Pandemia Covid-19 en una perspectiva comparada
}

Remi Castioni ${ }^{1}$

Adriana Almeida Sales de Melo $^{2}$

RESUMO: Investiga como as universidades brasileiras, espanholas e italianas mais bem situadas no ranking da Times Higher Education 2020, têm conduzido suas atividades durante a pandemia da Covid-19. A partir de pesquisa junto aos endereços eletrônicos das respectivas instituições e apoiados na literatura que analisa as mudanças que vêm ocorrendo no ensino superior, em perspectiva comparada, traça-se um panorama de como as universidades reagiram às restrições impostas pela pandemia. Um traço geral é que as universidades estrangeiras rapidamente migraram suas atividades de ensino para o modo remoto e no Brasil somente as estaduais paulistas o fizeram, enquanto as universidades federais paralisaram suas atividades de ensino no início da pandemia da COVID-19.

Palavras-chave: Covid-19. Governança. Universidades. Rankings Acadêmicos. Educação Comparada.

ABSTRACT: How Brazilian, Spanish and Italian universities better placed in the Times Higher Education Ranking 2020, conducted their activities during the Covid19 pandemic. Based on a web sites survey of the respective institutions and supported by the literature that analyzes the changes that have been occurring in higher education, in comparative perspective, an overview of how universities reacted to the restrictions imposed by the pandemic is outlined. A general trait is that foreign universities quickly migrated their teaching activities to the remote mode, while in Brazil only São Paulo's State Universities reacts, while federal universities paralyzed their teaching activities at the Covid-19 pandemic beginning.

Keywords: Covid-19. Governance. Universities. Academic Rankings. Comparative Education.

\footnotetext{
1 Universidade de Brasilia. E-mail: rcastioni@globo.com

2 Universidade de Brasilia. E-mail: adrianasalesdemelo@gmail.com
} 
RESUMEN: Cómo las universidades brasileñas, españolas e italianas mejor ubicadas en el ranking Times Higher Education 2020, llevan a cabo sus actividades durante la pandemia Covid-19. Basado en una encuesta de las direcciones de sitios electrónicos de las respectivas instituciones y respaldado por la literatura que analiza los cambios que han estado ocurriendo en la educación superior, en perspectiva comparada, se describe una visión general de cómo las universidades reaccionaron a las restricciones impuestas por la pandemia. Un rasgo general es que las universidades extranjeras migraron rápidamente sus actividades docentes al modo remoto, mientras que entre las brasileñas, sólo las estaduales de São Paulo lo hicieron, en cuanto que las universidades federales paralizaron sus actividades docentes durante el inicio de la Pandemia COVID-19.

Palabras clave: Covid-19. Gobernanza. Universidades. Rankings. Educación Comparada. 
As respostas que as Universidades estão construindo às demandas sociais e de ciência, tecnologia e inovação à Pandemia da Covid-19, revelam características de sua governança, adaptabilidade, otimização de recursos e de ativação rápida de recursos humanos e capacidade de pesquisa. A intensidade e a amplitude de tais respostas, com relação à investigação e às ações em tantas áreas do conhecimento; como a saúde pública, comunicação, construção de redes de solidariedade em diversas áreas, estudos urbanos e de produção de mercadorias, transporte e circulação; são exemplos do que as Universidades estão realizando, incitadas pelas múltiplas crises causadas pela Pandemia.

Como instituições que historicamente desenvolvem múltiplas ações consideradas estratégicas socialmente, as Universidades também respondem a partir de suas variadas competências (Sadlak, 2007). A complexidade da diversificação, do alcance e do ritmo próprio de cada uma delas, torna difícil a compreensão e a análise sobre a forma com que estão respondendo à Pandemia. Desde a inação, suspensão de atividades de ensino, pesquisa e extensão ao incentivo à geração de ações relativas à saúde pública, como a produção de álcoolgel até Equipamentos de Proteção Individual - EPIs para os profissionais que atuam na linha de frente; tais instituições reagiram, lastreadas em sua autonomia de governança e limitadas por seus recursos financeiros, de tempo e de capacidade de pessoas e grupos. Este momento histórico nos mostra o quanto tais instituições são importantes e conseguem interagir com as diversas demandas sociais advindas de problemas para os quais, até então, não havia parâmetros de design administrativo mundial para as respostas necessárias.

Sob a influência da New Public Management (NPM), ocorreu nos últimos 30 anos uma alteração sistemática da noção de governança da Administração Pública, ligada a um avanço na forma de governar (Osborne, 2006). Nos aspectos aplicados à universidade, os princípios da administração pública e de governança, abarcam hoje, segundo Capano (2011), quatro formas de organização da política do ensino superior: o hierárquico (hierarquical), o procedimental (procedural), a condução à distância (steering at the distance) e a auto governança (self-governance). Para cada uma delas há uma descrição das suas condicionalidades.

Diferentes instrumentos são utilizados para regular cada uma das referidas formas, que em geral podem ser assim classificadas: no procedimental, as instituições perseguem seus objetivos, mas os governos impõem as regras; na condução à distância, os governos assumem uma função regulatória, influenciando as instituições por meio de controles, propondo para tal, regras e incentivos; na auto governança, os governos se retiram de cena e intervém somente quando necessário. As universidades sempre foram instituições que reivindicaram 0 exercício da 
autonomia, pois têm a ver com sua feição e forma de organização (Balbachevsky \& Kohtamäki, 2019). Entretanto, em situações como as vivenciadas durante a Pandemia, sua reação inicial, ao mesmo tempo em que se caracterizou por dar visibilidade às ações já desenvolvidas por seus pesquisadores, nos campos das ciências da vida e da saúde, bem como algumas áreas tecnológicas, as demais áreas praticamente se fecharam em torno dos seus desígnios.

No Brasil, $95 \%$ da produção da pesquisa se realiza nas universidades públicas notadamente em 5 estaduais e 15 federais (Capes, 2018). Nessa direção é interessante destacar as duas principais frentes de exercício da autonomia: de um lado, as estaduais paulistas, que contam com total autonomia na gestão dos seus recursos e com uma poderosa agência de financiamento, a Fundação de Amparo à Pesquisa do Estado de São Paulo (FAPESP), com recursos superiores ao sistema federal, do outro lado, o Conselho Nacional de Desenvolvimento Científico e Tecnológico (CNPq). A FAPESP tem orçamento anual de $R \$ 1,6$ bilhão, contra $\mathrm{R} \$ 1,3$ bilhão do $\mathrm{CNPq}$ (dados do orçamento de 2020). Nessa perspectiva, as estaduais paulistas mantiveram atividades em quase todas as dimensões, já o sistema federal que segue um modelo excessivamente centralizado, praticamente paralisou suas atividades de ensino.

Assim como a crise do Estado de BemEstar social, a crise financeira de 2008 e tantas outras exigiram que novas respostas científicas e políticas fossem sendo construídas; a Pandemia da COVID-19 também está imprimindo novos rumos teóricos e epistemológicos à forma e à natureza como a administração pública busca entender as ações das instituições complexas como as Universidades. Uma nova agenda internacional está sendo atualmente construída a partir de tais respostas institucionais.

No sentido de apresentar um panorama das ações das Universidades relativas às suas respostas imediatas e iniciais de governança à Pandemia da Covid-19, este artigo se propõe a categorizar as ações principais que estão sendo realizadas nas melhores Universidades brasileiras, espanholas e italianas; a partir do reconhecimento público e acadêmico do ranking realizado pelo Times Higher Education (THE), intitulado World University Ranking (WUR) de 2020. A proposta de investigação se baseou na verificação das categorias fundamentais de construção do WUR: ensino, pesquisa, citações, respostas à indústria $e$ perspectiva internacional, bem como dos 13 indicadores que fazem parte de sua fundamentação metodológica.

Dessa forma, o texto a seguir está dividido em três partes, sendo a primeira sobre a metodologia de pesquisa que parte de uma perspectiva de análise comparada, tomando como foco a educação superior; a segunda parte sobre a importância dos rankings acadêmicos com relação à indução de melhoria da qualidade da educação superior e a terceira parte, que trata das ações das melhores Universidades brasileiras, espanholas e italianas, segundo as categorias centrais do WUR 2020, a partir das atividades 
divulgadas em seus endereços institucionais relativas ao enfrentamento da Pandemia da COVID-19.

\section{Educação Superior em perspectiva comparada}

As Universidades, segundo Philip Altbach (1998) se caracterizam no novo milênio como instituições que continuam sendo referência quanto ao seu sentido global e ao reconhecimento social de sua importância para o desenvolvimento nacional e internacional.

With its roots in medieval Europe, the modern University is at the centre of an international knowledge system that encompasses technology, communications and culture. (Altbach, 1998, p. xvii)

Sejam baseadas em variados modelos colegiados públicos ou nos diversos modelos de instituições privadas; sejam voltadas prioritariamente à pesquisa e à internacionalização; sejam voltadas ao progresso social ou incorporando todos estes objetivos; as Universidades contemporâneas primam cada vez mais por uma melhor governança. A partir de suas especificidades e culturas organizacionais próprias, a inserção cada vez mais profunda na inovação em todas as áreas do conhecimento torna-se uma necessidade. As demandas sociais se tornam cada vez mais presentes no direcionamento das pesquisas de base, assim como tais instituições se esforçam por construir conhecimento para dar suporte à formulação das políticas públicas locais e até mesmo internacionais.
Estudos estão sendo realizados no mundo inteiro, a partir das respostas dos sistemas nacionais e locais relativos às políticas educacionais e que acompanham também as respostas mais gerais dos países à Pandemia da COVID-19.

Após um primeiro momento de intenso isolamento social e suspensão das atividades escolares e acadêmicas presenciais, aos poucos pesquisadores e formuladores de políticas públicas se viram na obrigação de direcionar suas pesquisas para criar conhecimento sobre o tema em suas mais variadas formas.

Dar suporte científico às decisões políticas educacionais torna-se uma necessidade cada vez mais premente, gerando novas áreas de conhecimento $e$, na área da educação, vemos um retorno do estudo das evidências e da avaliação de políticas públicas, associadas às questões relacionadas à desigualdade social e acesso à educação.

Investigar como algumas Universidades atuaram para continuar realizando suas missões em tal momento histórico, numa perspectiva comparada, pode ajudar a construir novas estratégias políticas voltadas para a educação superior. O estudo dos rankings acadêmicos (Calderón \& França, 2018) associado às respostas das Universidades aos desafios de ação e 
formulação de políticas educacionais tem como objetivo melhorar nossa compreensão das boas práticas que já estão sendo realizadas como resposta rápida inicial para os desafios da Pandemia da COVID-19 vivenciados por tais instituições.

Em estudo sobre a reação dos países quanto às necessidades da educação pública, Reimers e Schleicher (2020) realizaram roteiro de pesquisa para tentar categorizar as múltiplas respostas nacionais à percepção dos desafios e suas respostas à sociedade.

Entre as preocupações, num primeiro grupo estariam as respostas relativas à necessidade do próprio reconhecimento da Pandemia da COVID-19 e suas consequências como emergência de saúde pública nacional, bem como sua amplitude. Para as instituições educacionais, seria o momento de estreitar as parcerias com os sistemas nacionais de saúde e suas redes de atuação, a fim de contribuir para a construção de protocolos e consensos sobre a suspensão de atividades e preservação de vidas. Internamente, tal momento inicial seria também voltado para a criação de novos órgãos colegiados com funções relativas ao acompanhamento das mudanças institucionais necessárias em tal momento histórico de emergência de saúde mundial.

Um segundo grupo de ações seria relativo às definições de princípios para redesenhar as estratégias necessárias ao enfrentamento, como: a proteção tanto do conjunto de funcionários e professores, quanto dos estudantes e suas famílias, a contribuição para a proteção da saúde e vida para toda a sociedade; assim como desenhar princípios para a proteção da saúde mental e emocional de todos; focando nas decisões colegiadas e considerando a escassez de tempo e recursos; sempre em diálogo com as instituições e autoridades de saúde pública local e internacional.

Um terceiro grupo de categorias a serem formuladas seria relativo aos aspectos didático-pedagógicos das respostas, no sentido de formular políticas públicas que permitam às instituições a reformulação de currículos, de calendário acadêmico, de regulamentação dos diversos aprendizados a distância, de forma remota, ou similares.

Em tal grupo de formulações estaria a preocupação com as ações relativas às formas de prover acesso, aparelhos de informática diversos e alternativas de conectividade, a partir de investigação sobre a necessidade de assistência dos estudantes, professores e demais funcionários das instituições. Decisões a serem tomadas em conjunto, não somente a partir da definição de novas regras e regulamentações, mas também a partir das necessidades e expectativas de todos; considerando o período necessário de isolamento ou distanciamento social assumido por cada país, assim como as necessidades institucionais de recuperação de tal período disruptivo.

O quarto grupo de preocupações que se coloca como prioridade entre os países pesquisados (Reimers \& Schleicher, 2020) se dirige à comunicação dos processos, à necessidade de criação de um planejamento comunicacional. Tais prioridades visam atingir a transparência e 
participação dos envolvidos no conjunto das estratégias, de todas as etapas de planejamento e decisão, assim como quanto a preocupação primordial tanto de esclarecer as ações institucionais que estão sendo construídas quanto de envolver todos os sujeitos nos processos, criando canais para que expressem suas participações.
Em quinto lugar, segundo as respostas dos países relacionadas na pesquisa, estão as categorias relativas ao acompanhamento $e$ avaliação permanente das ações e também envolvem tanto os diversos sujeitos quanto exigem uma modificação profunda da cultura organizacional das instituições, dos países e de suas políticas públicas.

\section{Sobre Benchmarking e Ranking: o que podemos aprender com tais ferramentas para a gestão pública da educação superior}

A questão da qualidade foi se tornando cada vez mais presente nas políticas educacionais desde os anos de 1980. Em conjunto com a universalização da educação e a melhoria da formação e do trabalho dos profissionais relacionados com a educação em todos os seus níveis, o tema da melhoria da qualidade se expandiu como um consenso, mesmo que também tenha ampliado sua polissemia, principalmente a partir do lançamento do programa de Educação para Todos da Organização das Nações Unidas (ONU) (Torres, 2001; Melo, 2004), que foi sendo implantado nos países com dinâmicas e abrangências diferentes, modificando a governança de tais políticas em favor da construção de uma agenda mundial.

Com relação à educação superior e à renovação da missão das suas instituições, a melhoria da qualidade tornou-se um objetivo a ser planejado, executado e especificamente regulado e avaliado. No bojo das questões relacionadas aos processos de globalização, a disputa pela captação de recursos entre as instituições públicas e privadas são necessidades que se expressam cada vez mais claramente, assim como 0 direcionamento das questões de governança.

Iniciativas como a Conferência Mundial sobre a Educação Superior em 1998 (Calderón, Pedro \& Vargas, 2011) e o próprio Processo de Bolonha, que teve suas raízes históricas ainda no século $X X$, com as discussões sobre a necessidade da criação de um Espaço Europeu de Educação Superior (Antunes, 2008), se juntaram às necessidades diversas dos países no sentido de buscar tal qualidade. $O$ aumento das possibilidades de geração de novos conhecimentos com a evolução das novas tecnologias relacionadas às ciências da computação e a forma como se deu a sua disseminação, ampliou as capacidades globais de comunicação, permitindo também a criação de novas formas de comparação entre as iniciativas de melhoria da qualidade da educação superior em todo o mundo. 
O que antes demandava imensos e custosos projetos de pesquisa para o levantamento primário de dados em cada país, tornou-se cada vez mais rápido e acessível, pois os próprios países e instituições foram construindo formas cada vez mais claras e transparentes de mostrar ao mundo inteiro as suas ações (Sadlak, 2007). As respostas aos financiamentos públicos, extrapolando a prestação de contas de recursos recebidos para a realização de políticas, programas e projetos, foi se tornando uma obrigação cotidiana e corriqueira, ampliando também a capacidade de melhoria dos processos de accountability em todo o mundo.

Se, desde os anos de 1970, os questionamentos do New Public Management impulsionaram as necessidades de melhoria da resposta institucional com relação à sociedade, no século XXI este movimento de melhoria da resposta pública tornou-se uma questão de sobrevivência para as instituições de educação superior. Além de acompanhar a legislação cada vez mais sofisticada das agências locais de reconhecimento, acreditação, avaliação e legislação em geral, tais instituições também tiveram que ampliar suas capacidades de competição num mundo cada vez mais globalizado.

A atração de novos alunos, a quantificação de produtos acadêmicos, a competição entre laboratórios e pesquisadores, a captação de recursos públicos e privados, a capacitação dos docentes e demais funcionários com relação às novas tecnologias e novas necessidades de divulgar melhor seu trabalho em todas as áreas; tudo isso - sem sublimar as respostas com relação aos grandes problemas sociais, como relacionados inicialmente - aumenta a responsabilidade das instituições de educação superior no mundo inteiro, especialmente no momento atual que exige respostas rápidas de diversas áreas de conhecimento para o enfrentamento da Pandemia da COVID19.

Todas as Universidades vivem num processo permanente de renovação, de repensar suas missões, de renovar seus objetivos, mesmo mantendo suas histórias e sua cultura organizacional. É um grande desafio para a burocracia universitária a sua renovação, a busca por novos caminhos e a atualização constante dos seus objetivos, especialmente com relação ao enfrentamento de uma pandemia.

Ainda que não haja imediatamente a criação ou mesmo a negociação relativa a uma nova agenda mundial para a educação superior pós-pandemia, muitas Universidades vão criando novos benchmarkings para tentar tornar mais eficientes e otimizados todos os esforços de seus agentes. Estabelecer novas metas internas colaborativas é um dos primeiros passos para tentar melhorar a resposta à pandemia. Como constataremos a seguir, as melhores Universidade são também as que estão atualmente estimulando as áreas do conhecimento a produzir respostas diferenciadas para a pandemia. $O$ incentivo à proliferação de atividades com a manutenção da qualidade da educação superior são desafios que vêm sendo incorporados no cotidiano das melhores Universidades. 
Os rankings e seus indicadores específicos também fazem parte da competição entre os países e instituições em suas mais variadas formas; desde a atração de recursos públicos e privados à atração de alunos para suas variadas áreas $e$ modalidades de ensino e pesquisa, sendo também consultados para verificar a melhoria das instituições a cada uma de suas publicações. Cada um deles funciona como regulamentação informal das performances, o que também gera críticas sobre a autonomia das instituições de educação superior para gerar seus próprios indicadores. Para o presente artigo nos baseamos no Worl University Ranking (WUR) 2020 da Times Higher Education (THE), especialmente considerando sua abrangência de 1.400 universidades localizadas em 92 países e sua metodologia, baseada em cinco categorias gerais e 13 indicadores de qualidade; além de acompanhar a declaração das instituições de como estão cumprindo os Objetivos para o Desenvolvimento Sustentável (ODS) da ONU.

Uma das categorias é relativa ao ensino de excelência, ao ambiente de aprendizagem em suas múltiplas modalidades, a partir de indicadores que vão desde um bom ambiente para a aprendizagem, passando pela relação entre quantidade de professores, alunos de graduação e doutorandos formados à reputação internacional dos seus professores.

A excelência em pesquisa também é uma das cinco grandes categorias, com indicadores que envolvem enquetes sobre a sua reputação, produtividade e produção dos grupos e pesquisadores. O impacto das citações relativo às publicações científicas é outra das ferramentas metodológicas principais, a fim de mostrar a influência internacional da pesquisa; aliado a outro grande tema que se refere à colaboração internacional entre redes e pesquisadores. A relação com a sociedade também é verificada através da transferência de conhecimento, produtos e patentes com as indústrias, assim como através da capacidade das instituições de realizar parcerias e atrair recursos da iniciativa privada.

A seguir, apresentamos um levantamento das principais ações que estão sendo desenvolvidas pelas principais Universidades brasileiras, italianas e espanholas, segundo o ranking THE-WUR 2020, como respostas imediatas à Pandemia da COVID-19, a partir de seus endereços eletrônicos de divulgação institucional. Para a classificação da condição "sem mudanças", utilizamos a observação anterior a decretação da Pandemia. 


\section{As Universidades brasileiras}

Com relação às Universidades brasileiras, selecionamos as 5 melhores no THE-WUR 2020: a Universidade de São Paulo (USP), na faixa dos 46,9-50,0 pontos; a Universidade de Campinas (UNICAMP), com 35,3-36,7 pontos e as seguintes com pontuação entre 28,3-35,2: a Universidade Federal de Minas Gerais (UFMG), a Universidade Federal do Rio Grande do Sul (UFRGS) e a Universidade Federal de Santa Catarina (UFSC) e chegamos às suas principais ações, que as destacam no universo de respostas acadêmicas à Pandemia, conforme dispostas no Quadro 1.

Com relação à USP, a pré-existência da maioria das atividades facilitou a rapidez das respostas, considerando a criação dos projetos do portal e-Aulas e repositório eDisciplinas virtuais; assim como a continuidade das defesas virtuais da pósgraduação. A criação de Rede USP para o diagnóstico da COVID-19, articulando as ações de todos os campi, bem como a criação de editais com recursos específicos para a pesquisa sobre diagnóstico, tratamento e vacina, demonstra a rapidez e eficiência das respostas à Pandemia; bem como a criação de start-ups específicas para incentivar a transferência de tecnologia, serviços e conhecimento para a área de saúde local, pública e privada, multiplicando as capacidades de resposta da instituição. A UNICAMP criou um Grupo de Trabalho COVID-19 com as Frentes de Divulgação, Articulação, Captação de Recursos e Tecnológica, com Frentes Interinstitucionais de Diagnóstico, Ensaios Clínicos, Engenharia Biomédica, Pesquisa e Desenvolvimento, Pesquisa Básica e Modelagem e Epidemiologia, assim como ampliou a capacidade de publicações para divulgação de atividades, bem como está estimulando novos tipos de publicação a partir de pré-print e early view; além da criação de possibilidades de atividades não-presenciais. As demais Universidades apresentaram suspensão de atividades e relatos de ações junto aos tradicionais parceiros das redes de saúde pública. 
Quadro 1 - Universidades brasileiras e atividades de enfrentamento da Pandemia da COVID19 em 2020 (mar-abril)

\begin{tabular}{|c|c|c|c|}
\hline Categorias & USP & UNICAMP & UFMG, UFGRS, UFSC \\
\hline Ensino & $\begin{array}{l}\text { Video-conferências; e- } \\
\text { Aulas; e-Disciplinas }\end{array}$ & $\begin{array}{l}\text { Adaptação de aulas para } \\
\text { atividades não- } \\
\text { presenciais }\end{array}$ & $\begin{array}{l}\text { Suspensão de aulas } \\
\text { presenciais }\end{array}$ \\
\hline Pesquisa & $\begin{array}{l}\text { Defesas remotas; criação } \\
\text { de Rede para o } \\
\text { Diagnóstico e Editais } \\
\text { específicos }\end{array}$ & $\begin{array}{l}\text { Defesas remotas; Grupo } \\
\text { de Trabalho COVID-19 }\end{array}$ & $\begin{array}{l}\text { Defesas remotas; } \\
\text { Comitês de } \\
\text { Enfrentamento à } \\
\text { Pandemia }\end{array}$ \\
\hline Citações & Incentivo à publicação & $\begin{array}{l}\text { Incentivo à publicação, ao } \\
\text { pre-print e early view }\end{array}$ & Sem mudanças \\
\hline $\begin{array}{l}\text { Influência } \\
\text { Internacional }\end{array}$ & $\begin{array}{l}\text { Criação de novas redes } \\
\text { de pesquisa e } \\
\text { comunicação }\end{array}$ & $\begin{array}{l}\text { Criação de novas redes } \\
\text { de pesquisa e } \\
\text { comunicação }\end{array}$ & Sem mudanças \\
\hline $\begin{array}{l}\text { Transferência de } \\
\text { Conhecimento }\end{array}$ & $\begin{array}{l}\text { Criação de start-ups, } \\
\text { produtos e serviços para } \\
\text { a rede de saúde local }\end{array}$ & $\begin{array}{l}\text { Plataformas de Pesquisa } \\
\text { e Solidariedade, Frente } \\
\text { de captação de recursos }\end{array}$ & $\begin{array}{l}\text { Feiras, Redes e } \\
\text { articulação local }\end{array}$ \\
\hline
\end{tabular}

Fonte: USP, 2020; Unicamp, 2020; UFMG, 2020; UFRGS, 2020; UFSC, 2020.

\section{As Universidades espanholas e italianas}

Escolhemos as universidades espanholas e italianas por abrigarem as mais antigas universidades do Mundo. Quanto às Universidades espanholas, as cinco melhores no THE-WUR 2020 são a Universitat Pompeu Fabra (UPF), com 57,8 pontos; a Universitat Autonoma de Barcelona (UAB), com 56,9 pontos; a Universitat de Barcelona (UB), na faixa dos $50,1-53,7$ pontos, todas públicas e localizadas na Região Autônoma da Catalunha; a Universidad Autònoma de Madrid (UAM) e a Universidad de Navarra (UNAV) esta, particular e multicampi, ligada a obra da Opus Dei da igreja católica, ambas na faixa dos 46,9-50,0 pontos. Suas principais ações estão explicitadas no Quadro 2.
Assim como as melhores Universidades brasileiras, as melhores Universidades espanholas também se movimentaram para dar respostas rápidas às necessidades acadêmicas e sociais relativas à Pandemia. Todas as citadas elaboraram suas normas para regulamentar o ensino remoto, assim como as defesas de trabalhos acadêmicos de graduação e pós-graduação, com destaque para a UPF e a UB, que divulgam sua preocupação com a interlocução com os campos de estágio públicos e privados, de forma a garantir sua continuidade. 
Quadro 2 - Universidades espanholas e atividades de enfrentamento da Pandemia da COVID19 em 2020 (mar-abril)

\begin{tabular}{|c|c|c|c|c|c|}
\hline Categorias & UPF & UAB & UB & UAM & UNAV \\
\hline Ensino & $\begin{array}{l}\text { Remoto, com } \\
\text { articulação } \\
\text { com empresas } \\
\text { de estágio }\end{array}$ & $\begin{array}{l}\text { Remoto, com } \\
\text { atenção para } \\
\text { os alunos em } \\
\text { mobilidade } \\
\text { acadêmica }\end{array}$ & $\begin{array}{l}\text { Remoto, com } \\
\text { manutenção } \\
\text { dos estágios }\end{array}$ & Remoto & Remoto \\
\hline Pesquisa & $\begin{array}{l}\text { Defesas } \\
\text { remotas; } \\
\text { estímulo a } \\
\text { grupos de } \\
\text { pesquisa e } \\
\text { laboratórios }\end{array}$ & $\begin{array}{l}\text { Defesas } \\
\text { remotas; } \\
\text { estímulo a } \\
\text { grupos de } \\
\text { pesquisa e } \\
\text { laboratórios }\end{array}$ & $\begin{array}{l}\text { Defesas } \\
\text { remotas, } \\
\text { estímulo a } \\
\text { grupos de } \\
\text { pesquisa e } \\
\text { laboratórios }\end{array}$ & $\begin{array}{l}\text { Defesas } \\
\text { remotas }\end{array}$ & $\begin{array}{l}\text { Defesas } \\
\text { remotas }\end{array}$ \\
\hline Citações & $\begin{array}{l}\text { Sem } \\
\text { mudanças }\end{array}$ & $\begin{array}{l}\text { Sem } \\
\text { mudanças }\end{array}$ & $\begin{array}{l}\text { Sem } \\
\text { mudanças }\end{array}$ & $\begin{array}{l}\text { Sem } \\
\text { mudanças }\end{array}$ & $\begin{array}{l}\text { Sem } \\
\text { mudanças }\end{array}$ \\
\hline $\begin{array}{l}\text { Influência } \\
\text { Internacional }\end{array}$ & $\begin{array}{l}\text { Sem } \\
\text { mudanças }\end{array}$ & $\begin{array}{l}\text { Criação de } \\
\text { novas redes de } \\
\text { pesquisa e } \\
\text { comunicação }\end{array}$ & $\begin{array}{l}\text { Sem } \\
\text { mudanças }\end{array}$ & $\begin{array}{l}\text { Sem } \\
\text { mudanças }\end{array}$ & $\begin{array}{l}\text { Articulação } \\
\text { com grupos de } \\
\text { pesquisa } \\
\text { internacionais }\end{array}$ \\
\hline $\begin{array}{l}\text { Transferência } \\
\text { de } \\
\text { Conhecimento }\end{array}$ & $\begin{array}{l}\text { Criação de } \\
\text { crowfundings } \\
\text { relativos à } \\
\text { Pandemia }\end{array}$ & $\begin{array}{l}\text { Integração de } \\
\text { grupos de } \\
\text { pesquisa com } \\
\text { a Generalitat } \\
\text { de Cataluña; } \\
\text { criação de } \\
\text { portais: UAB } \\
\text { responde e } \\
\text { Colaboração } \\
\text { com a Mídia }\end{array}$ & $\begin{array}{l}\text { Criação de } \\
\text { canal } \\
\text { ReflexionsUB } \\
\text { de opiniões de } \\
\text { pesquisadores } \\
\text { sobre a } \\
\text { Pandemia }\end{array}$ & $\begin{array}{l}\text { Sem } \\
\text { mudanças }\end{array}$ & $\begin{array}{l}\text { Integração } \\
\text { com grupos de } \\
\text { pesquisa } \\
\text { nacionais e } \\
\text { internacionais } \\
\text { para criação } \\
\text { de rede de } \\
\text { investigação }\end{array}$ \\
\hline
\end{tabular}

Fonte: UPF, 2020; UAB, 2020; UB, 2020; UAM, 2020; UNAV, 2020.

Com relação à pesquisa, detectamos em seus endereços institucionais especialmente a divulgação dos grupos de pesquisa e laboratórios que estão desenvolvendo atividades relativas à Pandemia.

Com relação à transferência de conhecimento, percebemos na UAB uma importante iniciativa tanto de criação de portais de comunicação com a sociedade, quanto com relação à integração de grupos de pesquisa à Generalitat da Cataluña (governo regional) com a cessão de profissionais de saúde e fabricação de produtos necessários para $\mathrm{O}$ enfrentamento da Pandemia.

Dentre as Universidades italianas, as melhores segundo o THE-WUR 2020 são a Sant'Anna Scuola Universitaria Superiore - Pisa (SASUS-PISA), com 57,3 pontos; a Scuola Normale Superiore di Pisa (SNS-PISA), com 57,2 pontos; a Università di Bologna (UNIBO), com 56 pontos; a Università Degli Studi de Padova (UNIPD) e a Università Vita-Sallute San Raffaele (UNISR), ambas com pontuação 
entre 50,1-53,7. Esta última privada e localizada no epicentro da pandemia do norte da Itália, a região da Lombardia. As ações das universidades italianas estão organizadas no Quadro 3.

As respostas das Universidades italianas selecionadas também demonstram os caminhos percorridos na adaptação às dificuldades relativas ao início da Pandemia da COVID-19, tanto quanto revelam suas capacidades já instaladas e recém construídas, num momento em que já se pensa em toda a Europa nos calendários e cronogramas para a retomada das atividades presenciais ou semipresenciais nas instituições de ensino superior.

Quanto ao ensino, a modalidade a distância foi instalada em todas as Universidades europeias investigadas, com tipos diferentes de avaliação remota para viabilizar o encerramento do ano acadêmico de 2019/20. Quanto às demais categorias, merece destaque a atuação da SASUS-PISA com relação à interação de pesquisa sobre robótica e auxílio local com relação à desinfecção sanitária de ambientes de atendimento à saúde.

Quadro 3 - Universidades italianas e atividades de enfrentamento da Pandemia da COVID-19 em 2020 (mar-abril)

\begin{tabular}{|c|c|c|c|}
\hline Categorias & SASUS-PISA & $\begin{array}{l}\text { SNS-PISA, UNIBO, } \\
\text { UNIPD, }\end{array}$ & UNISR \\
\hline Ensino & $\begin{array}{l}\text { Remoto, com criação de } \\
\text { novos canais de ensino } \\
\text { e aprendizagem }\end{array}$ & Remoto & Remoto \\
\hline Pesquisa & $\begin{array}{l}\text { Defesas remotas; } \\
\text { estímulo a grupos de } \\
\text { pesquisa e laboratórios }\end{array}$ & $\begin{array}{l}\text { Defesas remotas; } \\
\text { estímulo a grupos de } \\
\text { pesquisa e laboratórios }\end{array}$ & Sem mudanças \\
\hline Citações & Sem mudanças & Sem mudanças & Sem mudanças \\
\hline $\begin{array}{l}\text { Influência } \\
\text { Internacional }\end{array}$ & $\begin{array}{l}\text { Articulação com grupos } \\
\text { de pesquisa } \\
\text { internacionais }\end{array}$ & Sem mudanças & Sem mudanças \\
\hline $\begin{array}{l}\text { Transferência de } \\
\text { conhecimento }\end{array}$ & $\begin{array}{l}\text { Criação cooperativa de } \\
\text { produtos de Robótica }\end{array}$ & $\begin{array}{l}\text { Realização de webinars } \\
\text { e colaboração com } \\
\text { grupos de pesquisa } \\
\text { locais }\end{array}$ & Sem mudanças \\
\hline
\end{tabular}

Fonte: SantannaPisa, 2020; SNS-PISA, 2020; UNIBO 2020; UNIPD, 2020; UNISR, 2020.

\section{Breves Considerações}

Um traço marcante entre as universidades brasileiras e as europeias deu-se notadamente nas atividades de ensino. Enquanto as estrangeiras analisadas reposicionaram suas atividades de ensino no modo on-line, se utilizando das tecnologias existentes como Moodle, Google Clasroom e transmitindo aulas ou realizando webinários via Zoom, Google Meet ou Teams, ou outras plataformas, as 
brasileiras se fecharam, com exceção das paulistas. As plataformas de aprendizagem de tais empresas tiveram visibilidade para além das previsões dos seus planos de negócios a ponto de liberarem gratuitamente muitas das suas funcionalidades.

A campanha lançada durante a Pandemia pela UNESCO e denominada de learning never stops, pode ter repercutido na decisão do Conselho Nacional de Educação (CNE, 2020) no Brasil de reconhecer o uso das plataformas de aprendizagem para todas as etapas e modalidades de educação e pode vir a provocar mudanças importantes na oferta de ensino superior, onde $1 / 3$ dos ingressos já ocorre por meio da modalidade EaD. É curioso que as universidades federais que tiveram recentemente um plano de expansão na sua infraestrutura com o Programa do Governo Federal de Apoio a Planos de Reestruturação e Expansão das Universidades Federais (REUNI) e a criação da Universidade Aberta do Brasil (UAB), não tenham superado esta dicotomia entre ensino presencial e ensino a distância, quando os sistemas mais avançados do mundo já caminham para uma educação híbrida.

Uma ação mais centrada no desenvolvimento das competências e da autonomia do estudante já era uma realidade antes e agora é uma necessidade. É preciso vencer resistências e atrasos, tanto de ordem infraestrutural como de conceito com relação às missões das universidades. As melhores universidades do mundo estão enfrentando a Pandemia da COVID-19 com respostas rápidas no âmbito do ensino, da pesquisa $\mathrm{e}$ do diálogo internacional em sintonia com as demandas da sociedade.

Recebido em 23 de julho de 2020 Aprovado em 02 de setembro de 2020 
Altbach, P. (1998). Comparative Higher Education: knowledge, the University and development. WESTPORT: Ablex Publishing.

Antunes, F. (2008). O espaço europeu de ensino superior para uma nova ordem educacional?. ETD - Educação Temática Digital, 9, 1-28. https://doi.org/10.20396/etd.v9in.esp.725

Balbachevsky, E., \& Kohtamäki, V. (2019). Autonomia e Governança Universitária: uma abordagem comparativa USP (Brasil) e UTA (Finlândia). In: Marcovitch, J., et al (org.). Repensar a Universidade: Impactos para a Sociedade. São Paulo: Com-Arte/FAPESP. P. 235-48.

Calderón, A. I., \& França, C. M. (2018) Rankings acadêmicos na educação superior: tendências da literatura ibero-americana. Avaliação: revista da avaliação da educação superior, Sorocaba, v. 23, p. 448-466.

Calderón, A. I., Pedro, R. F., \& Vargas, M. C. (2011). Responsabilidade social da educação superior: a metamorfose do discurso da UNESCO em foco. Revista Interface Comunicação, Saúde, Educação. V.15, n.39, p.1.185-98, out-dez.

Capano, G. (2011). Government continues to do its job. A comparative study of governance shifts in the higher education sector. Public Administration, v. 89, n. 4, p. 1622-1642.

CAPES (2020). Research in Brazil. A report for CAPES by Clarivate Analytics. Brasília: Clarivate Analytics. Disponível em: https://bityli.com/ibJuX. Publicado em 01/2018. Acesso em: 30 abr. 2020.

CNE/MEC. (2020). Reorganização do Calendário Escolar e da possibilidade de cômputo de atividades não presenciais para fins de cumprimento da carga horária mínima anual, em razão da Pandemia da COVID-19. Parecer N. 5/2020, aprovado em 28 abr. 2020. Brasília: CNE. Disponível em: https://bityli.com/xOlUi.

Melo, A. A. S. (2004). A mundialização da educação. Maceió: Edufal.

Osborne, S. P (2006). The New Public Governance? Public Management Review, v. 8, n. 3 , p. 377-387.

Reimers, F. M., \& Schleicher, A. (2020). A framework to guide an educational response to the COVID-19 Pandemic of 2020. OECD.

Sadlak, J. (2007). Why higher education is changing - Implications for access, governance, quality evaluation and funding. The Council of Higher Education, Ankara: Binkent University.

SantannaPisa (2020). Endereço eletrônico. https://www.santannapisa.ittit. Acesso em: 15 mai.

SNS (2020). Endereço eletrônico. https://www.sns.it/. Acesso em: 15 mai.

Torres, R. M. (2001). Educação para todos, a tarefa por fazer. São Paulo: Artmed.

UAB (2020). Endereço eletrônico. https://www.uab.cat/. Acesso em: 15 mai. 
UAM (2020). Endereço eletrônico. https://www.uam.es. Acesso em: 15 mai.

UB (2020). Endereço eletrônico. https://www.ub.edu/web/ub/ca/. Acesso em: 15 mai.

UFMG (2020). Endereço eletrônico. https://ufmg.br/. Acesso em: 15 mai.

UFRGS (2020). Endereço eletrônico. http://www.ufrgs.br/ufrgs/inicial. Acesso em: 15 mai.

UFSC (2020). Endereço eletrônico. https://ufsc.br/. Acesso em: 15 mai.

UNAV (2020). Endereço eletrônico. https://www.unav.edu/. Acesso em: 15 mai

UNIBO (2020). Endereço eletrônico. https://www.unibo.it/it. Acesso em: 15 mai.

Unicamp (2020). Endereço eletrônico. https://www.unicamp.br/unicamp/. Acesso em: 15 mai.

UNIPD (2020). Endereço eletrônico. https://www.unipd.it/. Acesso em: 15 mai.

UNISR (2020). Endereço eletrônico. https://www.unisr.it/homepage. Acesso em: 15 mai.

UPF (2020). Endereço eletrônico. https://www.upf.edu/inicio. Acesso em: 15 mai.

USP (2020). Endereço eletrônico. https://www5.usp.br/. Acesso em: 15 mai. 